\title{
A Tunable WDM Wavelength Converter Based on Cross-Phase Modulation Effects in Normal Dispersion Holey Fiber
}

\author{
Ju Han Lee, Zulfadzli Yusoff, Walter Belardi, Morten Ibsen, Tanya M. Monro, and David J. Richardson
}

\begin{abstract}
We demonstrate a tunable wavelength-division-multiplexing wavelength converter based on cross-phase modulation in just $5.8 \mathrm{~m}$ of highly nonlinear normal dispersion holey fiber (HF) and incorporating a precise compression-tuned apodized fiber-grating filter. We achieve both error-free and penalty-free $10-\mathrm{Gb} / \mathrm{s}$ wavelength conversion over a $\sim 15-\mathrm{nm}$ bandwidth. The short device length and correspondingly reduced pulse walkoff effect ensures a constant wavelength-converted signal pulsewidth across the full tuning range. The use of $\mathrm{HF}$ with normal dispersion eliminates the coherence-degradation related intensity noise previously observed in experiments employing anomalously dispersive HF.
\end{abstract}

Index Terms-Gratings, nonlinear optics, optical fiber communication, optical fiber devices, optical fiber switches.

\section{INTRODUCTION}

$\mathbf{R}$ ECENT PROGRESS in the design and fabrication of holey fiber (HF) has resulted in widespread interest in the use of such technology for a variety of fiber-optic communication and photonics devices and applications [1], [2]. The strong wavelength dependence of the effective index between the core and the cladding, and the possibility of large effective-index contrasts between the core and cladding in HF leads to a broader range of optical properties in these fibers than are realizable using conventional fiber fabrication approaches. HFs can have a much higher nonlinearity per unit length than conventional fibers, and consequently devices based on such fibers can be much shorter in length and/or operate at lower power levels than their conventional counterparts. For example, we have demonstrated a regenerative all-optical switch based on self-phase modulation (SPM) and spectral filtering in just $3.3 \mathrm{~m}$ of HF [1]. We also showed that it was possible to obtain $\sim 42$-dB signal gain in an $L^{+}$-band Raman amplifier based on just 75-m length of HF [2]. Sharping et al. reported results on the operation of a nonlinear optical loop mirror switch based on cross-phase modulation (XPM) in a 5.8-m HF [3]. Recently, we demonstrated that HF technology could be applied to provide another important nonlinear function required within wavelength-division-multiplexing (WDM) systems-namely tunable WDM wavelength conversion [4], [5]. Multiple wavelength conversion was readily achieved at a data rate of $10 \mathrm{~Gb} / \mathrm{s}$

Manuscript received August 26, 2002; revised December 2, 2002

The authors are with the Optoelectronics Research Centre, University of Southampton, Highfield, Southampton, SO17 1BJ, U.K. (e-mail: jhl@orc.soton.ac.uk).

Digital Object Identifier 10.1109/LPT.2002.807927

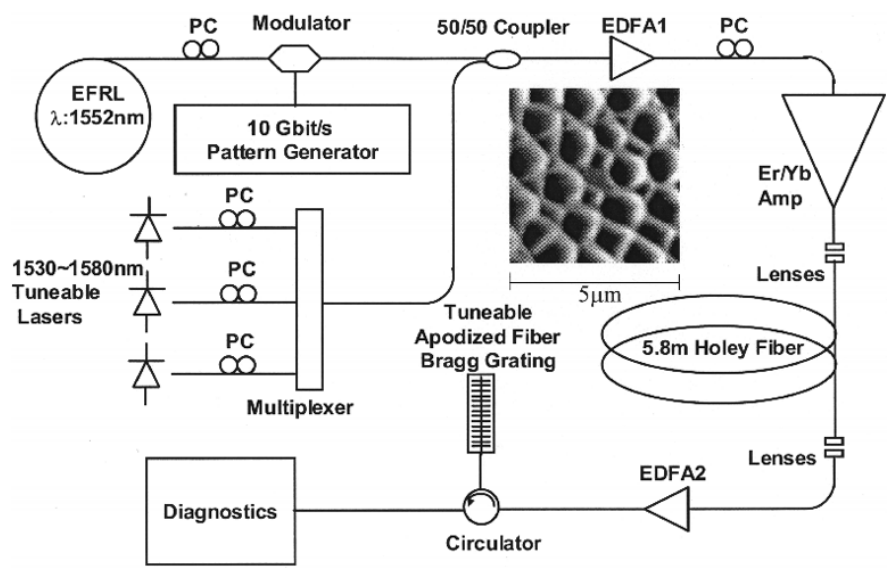

Fig. 1. Experimental setup. Inset: Cross-sectional SEM image of the normal dispersion HF used within these experiments.

using either XPM effect [4] or four-wave mixing (FWM) effect [5]. However, in the previous wavelength-conversion experiments based on XPM and passive filtering, we observed a degree of amplitude noise on the converted signals which we attributed to coherence degradation due to the anomalous dispersion of the HF used [4]. The amplitude noise resulted in a 4-dB power penalty and an error floor at the $10^{-9}$ bit-error-rate (BER) level relative to that of the soliton control pulses.

In this letter, we demonstrate a high-performance tunable WDM wavelength converter based on HF with normal dispersion. We demonstrate multiple wavelength conversion over a $\sim 15$-nm bandwidth at a data rate of $10 \mathrm{~Gb} / \mathrm{s}$ using a combination of XPM in a short length of HF and narrow-band spectral filtering. Error-free, penalty-free wavelength conversion performance is obtained and the amplitude noise previously observed is effectively eliminated. As well as demonstrating a much shorter device than previously reported wavelength conversion based on the XPM and filtering [6], [7], we also demonstrate here the use of a compression-tuned narrow-band apodized fiber Bragg grating (FBG) filter to obtain accurate high-quality spectral definition of the wavelength-converted signal. The use of an apodized grating filter allows for excellent extinction of the associated residual continuous-wave $(\mathrm{CW})$ radiation, pump beams, and adjacent wavelength-converted channels.

\section{EXPERIMENTAL RESULTS}

Our experimental set up is shown in Fig. 1. 2.5-ps pulses at a repetition rate of $10 \mathrm{GHz}$ are first generated using a 


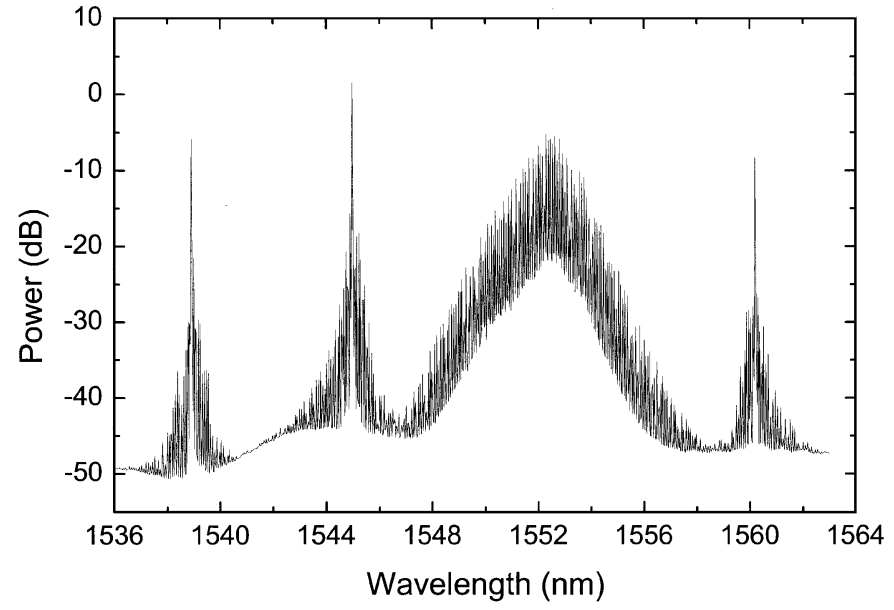

Fig. 2. Measured optical spectrum of the three wavelength-converted signals and of the control pulses after the HF.

regeneratively mode-locked erbium fiber ring laser (EFRL) operating at a wavelength of $1552 \mathrm{~nm}$. These pulses are then modulated to obtain a $10-\mathrm{Gb} / \mathrm{s} 2^{15}$-pseudorandom control pulse stream. We combined the control pulses and three probe beams together using a combination of 50/50 couplers prior to launching the light into the HF. The probe beams were generated from $\mathrm{CW}$ external cavity lasers tunable in the range 1530-1580 nm. Polarization controllers were included on both the control and probe launching paths into the HF so that all of the beams could be launched onto a single polarization axis of the polarization-maintaining HF. Both the control and probe signals were amplified using an $\mathrm{Er}-\mathrm{Yb}$ amplifier with a maximum saturated output power of $\sim 800 \mathrm{~mW}$, and were then lens coupled into a 5.8-m length of HF with a coupling efficiency of $30 \%$. A cross-sectional scanning electron microscope (SEM) profile of the highly nonlinear normal dispersion HF used in this experiment is shown in the inset of Fig. 1. The core is elliptical in shape (axis ratio of $\sim 1.5$ ) with a minimum axis dimension of $\sim 1.0 \mu \mathrm{m}$. The outer diameter of the HF is $100 \mu \mathrm{m}$. The measured dispersion at the wavelength of $1550 \mathrm{~nm}$ was $\sim-350 \mathrm{ps} / \mathrm{nm}-\mathrm{km}$ and the measured loss was $300 \mathrm{~dB} / \mathrm{km}$. From a measurement of the SPM-induced nonlinear phase shift versus launched optical power, we obtained an estimated value of $\gamma=\sim 60 \mathrm{~W}^{-1} \cdot \mathrm{km}^{-1}$ for the nonlinearity coefficient corresponding to an estimated effective area of $A_{\text {eff }}=1.5( \pm 0.2) \mu \mathrm{m}^{2}$. This nonlinearity is $\sim 30$ times higher than that of a conventional dispersion-shifted fiber.

XPM between the control signal and the $\mathrm{CW}$ beams results in chirping of the $\mathrm{CW}$ laser beam where these beams overlap temporally within the fiber. This frequency chirping can then be converted to a frequency converted signal by passing this chirped signal through a narrow-band filter which serves to eliminate the residual unchirped $\mathrm{CW}$ signal as well as to select one of the two XPM-induced side bands [7]. The measured output spectrum from the HF is shown in Fig. 2. It is clearly evident that each probe beam is spectrally broadened due to XPM induced by the control signal. The control signal itself also shows a small amount of spectral broadening caused by SPM. XPM among three CWs and SPM of each CW could be generated in some degree. However, the corresponding

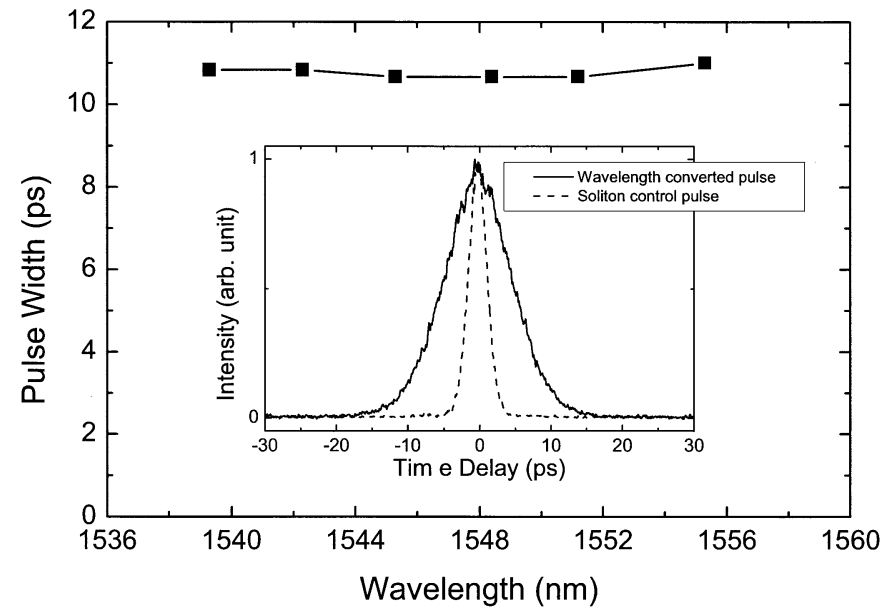

(a)

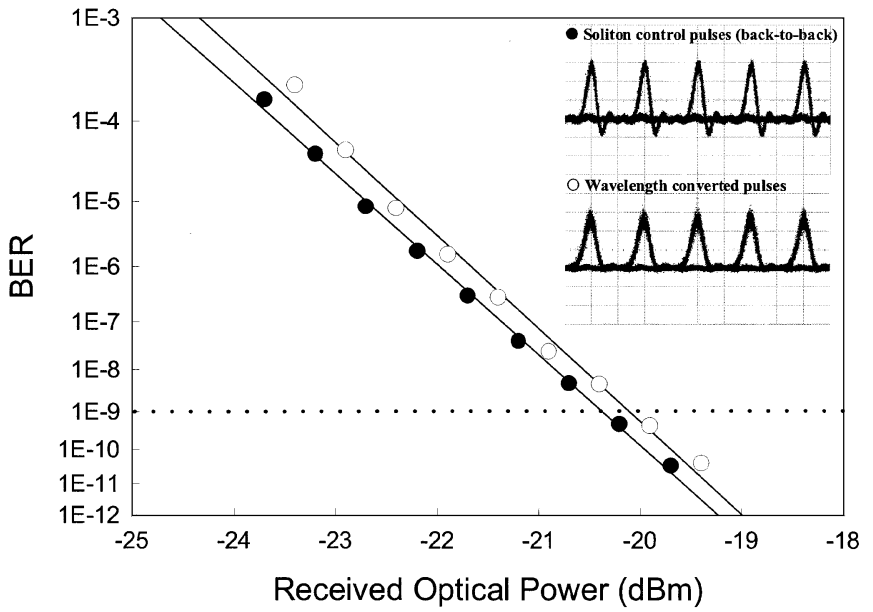

(b)

Fig. 3. (a) Measured full-width at half-maximum of the wavelength-converted pulses as a function of the probe beam wavelength for the case of wavelength conversion of a single channel. The inset shows the SHG autocorrelation traces of the wavelength-converted pulses at $1545 \mathrm{~nm}$, and the input pulses. (b) Measured BER versus received optical power for wavelength conversion of $10-\mathrm{Gb} / \mathrm{s}$ data pulses to a wavelength of $1545 \mathrm{~nm}$.

frequency chirping would be very small compared to that from XPM between the CWs and pump due to the lower peak powers of the $\mathrm{CW}$ pumps. In our experiment, we used an apodized FBG for the spectrum-slicing filter. The filter has a $3-\mathrm{dB}$ bandwidth of $0.55 \mathrm{~nm}$ and a $30-\mathrm{dB}$ bandwidth of $1.5 \mathrm{~nm}$. The FBG was embedded in a layered flexible plate that, when bent, allowed for 15-nm wavelength tuning of the filter central wavelength due to the resulting compressive strain applied to the FBG [8]. The FBG provides both high precision and high efficiency spectral slicing as well as significant suppression of all residual CW components, pump beams, and adjacent wavelength channels. The particular FBG filter is more fully described in [4].

Initially, we characterized the performance of our wavelength converter in the temporal domain using a second harmonic generation (SHG) autocorrelator $(<100-\mathrm{fs}$ resolution). The measured temporal width of the wavelength-converted pulses as a function of probe-beam wavelength is plotted in Fig. 3(a). The pulsewidths of the converted pulses were observed to be almost constant at $\sim 11 \mathrm{ps}$ over the full $\sim 15$-nm wavelength 
tuning wavelength range of our FBG. The walkoff between pump and signal was indeed quite significant and would have generated uneven output pulsewidths over the 15-nm tuning range without bandwidth reduction. However, the use of a narrow bandpass filter $(0.5-\mathrm{nm} 3-\mathrm{dB}$ bandwidth) restricted the output signal spectrum such that the temporal pulse shape was almost entirely determined by the filtered spectral bandwidth. The time-bandwidth product of the converted pulses was $\sim 0.5$ which, although somewhat larger than that of the original control soliton pulses ( 0.32 , transform-limited), still indicates high-quality pulses. The amplitude noise observed on the converted pulses in our previous experiments, which can be attributed to coherence degradation due to the anomalous dispersion of the particular HF used [9], was eliminated as we anticipated in our previous paper [4] [see the eye diagrams of Fig. 3(b)]. A detailed numerical study on the coherence-degradation problem associated with dispersion in $\mathrm{HF}$ could be found from the recent work by Dudley et al. [10]. We performed BER measurements on one channel at a wavelength of $1545 \mathrm{~nm}$. Fig. 3(b) shows both the measured BER and eye diagrams for the soliton control and wavelength-converted 10-Gb/s pulses. We obtained error-free, almost penalty-free $(\sim 0.5-\mathrm{dB}$ power penalty), wavelength conversion performance. Compared to the performance of the highly nonlinear fiber-based device in [6], we can see a benefit of using HF in terms of length/power product since our result shows a lower value of $1.2(\mathrm{~m} \cdot \mathrm{W})$ than the $10(\mathrm{~m} \cdot \mathrm{W})$ reported in [6]. Both of the results show output pulsewidths that are almost constant over the device operating bandwidth, although relatively broader output temporal pulsewidths are observed in our HF-based wavelength converter.

\section{CONCLUSION}

We have experimentally demonstrated a high-performance HF-based WDM wavelength converter using XPM and spectral filtering. Error-free, penalty-free multiple wavelength conversion of $10-\mathrm{Gb} / \mathrm{s}$ return-to-zero data pulses over a $\sim 15-\mathrm{nm}$ bandwidth was reliably obtained using a combination of just $5.8 \mathrm{~m}$ of highly nonlinear, normally dispersive HF and an apodized FBG filter. Use of an apodized grating filter with high extinction ratio allows for excellent suppression of residual $\mathrm{CW}$ light, pump beams, and adjacent wavelength-converted channels. The use of a short length of high nonlinearity HF with normal dispersion eliminates coherence-degradation effects and reduces walkoff effects. High-performance WDM wavelength conversion is thus obtained. Ultimately, HF technology should prove to be a powerful way to realize a wide range of practical nonlinear optical devices for fiber-optic communication systems.

\section{ACKNOWLEDGMENT}

The authors would like to thank M. R. Mokhtar and C. S. Goh for the use of their FBG and related help.

\section{REFERENCES}

[1] P. Petropoulos, T. M. Monro, W. Belardi, K. Frusawa, J. H. Lee, and D. J. Richardson, "2 R-regenerative all-optical switch based on a highly nonlinear holey fiber," Opt. Lett., vol. 26, pp. 1233-1235, 2001.

[2] Z. Yusoff, J. H. Lee, W. Belardi, T. M. Monro, P. C. Teh, and D. J. Richardson, "Raman effects in a highly nonlinear holey fiber: Amplification and modulation," Opt. Lett., vol. 27, pp. 424-426, 2002.

[3] J. E. Sharping, M. Fiorentino, P. Kumar, and R. S. Windeler, "All-optical switching based on cross-phase modulation in microstructure fiber," IEEE Photon. Technol. Lett., vol. 14, pp. 77-79, Jan. 2002.

[4] J. H. Lee, Z. Yusoff, W. Belardi, M. Ibsen, T. M. Monro, B. Thomsen, and D. J. Richardson, "A holey fiber based WDM wavelength converter incorporating an apodized fiber Bragg grating filter," presented at the Proc. CLEO 2002, Long Beach, CA, May 2002, Postdeadline Paper CPDB5.

[5] J. H. Lee, W. Belardi, K. Furusawa, P. Petropoulos, Z. Yusoff, T. M. Monro, and D. J. Richardson, "Four-wave mixing based 10-Gb/s tunable wavelength conversion using a holey fiber with a high SBS threshold," IEEE Photon. Technol. Lett., vol. 15, pp. 440-442, Mar. 2003.

[6] J. Yu and P. Jeppesen, "80-Gb/s wavelength conversion based on crossphase modulation in high-nonlinearity dispersion-shifted fiber and optical filtering," IEEE Photon. Technol. Lett., vol. 13, pp. 833-835, Aug. 2001

[7] P. Ohlen, B.-E. Olsson, and D. Blumenthal, "Wavelength dependence and power requirements of a wavelength converter based on XPM in a dispersion-shifted optical fiber," IEEE Photon. Technol. Lett., vol. 12, pp. 522-524, May 2000.

[8] S. Y. Set, B. Dabarsyah, C. S. Goh, K. Katoh, Y. Takushima, K. Kikuchi, Y. Okabe, and N. Takeda, "A widely tunable fiber Bragg grating with a wavelength tenability over $40 \mathrm{~nm}$," presented at the OFC 2001, Anaheim, CA, Mar. 2001, Paper MC4.

[9] N. Nakazawa, H. Kubota, and K. Tamura, "Random evolution and coherence degradation of a high-order optical soliton train in the presence of noise," Opt. Lett., vol. 24, pp. 318-320, 1999.

[10] J. M. Dudley and S. Coen, "Numerical simulations and coherence properties of supercontinuum generation in photonic crystal and tapered optical fibers," IEEE J. Select. Topics Quantum Eletron., vol. 8, pp. 651-659, May/June 2002. 